\title{
The relation between mathematical object/mathematical name: Conceptual changes between designation, description, denomination and definition.
}

\author{
Giorgio Bolondi ${ }^{1}$, Federica Ferretti ${ }^{1}$, and Alessandro Gambini ${ }^{2}$ \\ ${ }^{1}$ Department of Mathematics, Alma Mater Studiorum University of Bologna, Bologna, Italy \\ 2. Department of Mathematics, University of Ferrara. Ferrara, Italy \\ For correspondence: federica.ferretti5@gmail.com
}

\begin{abstract}
The actions of designation, description, denotation, denomination and definition are crucial in the didactic activity in the classroom (D'Amore and Fandiño Pinilla, 2012) since they embody different interplays between objects, representations, properties, names (in the sense of Duval (2008)). Switching from one action to the other may be the result of a conceptual change (diSessa, 2006). We present the result of a teaching experiment in classes of grades from 2 to 4 where the relation object/name is investigated in the case of the circle. The experiment makes use of a particular artefact, the Lénárt Spheres (Lénárt, 1996). Comparative geometry activities allow to deal with geometrical objects in a learning environment where the relations between objects, representations and properties are different from the usual ones, hence implying a restructuration of the interplays between them. As a result of the teaching experiment, as can be seen, in particular, from the comparison of initial and conclusive questionnaires, children started a change of their way of associating a name to an object. We argue that this is due also to a conceptual change and not only to "learning what was taught".
\end{abstract}

Keywords: Conceptual change, Lénárt Spheres, designation, definition, description.

\section{The theoretical frameworks, the methodology and the research questions}

\section{Mathematical objects and mathematical names in the classroom}

Several ways for associating a mathematical name to a mathematical object (and vice versa) are daily involved in the didactic activity of the teachers and in the learning processes of students. Examples of these actions are designation, description, denotation, denomination and definition (D'Amore and Fandiño Pinilla, 2012). These actions are epistemologically and cognitively different and they embody different possible interplays between objects, representations, properties, characteristics and names (see Bolondi et al, 2014). In fact, these actions are in the semiotic core of the teaching-learning process (see f.i. Duval, 2008, but also Radford, 2003). It is natural to consider the definition as a final and adequate way for expressing the pairings names/objects; it is in fact a typical form of advanced mathematical thinking.

A description of some of these interplays, which makes explicit the discussion of D'Amore and Fandiño Pinilla (2012), has been proposed in Bolondi et al (2014), and can be synthesized in this diagram in Figure 1. 


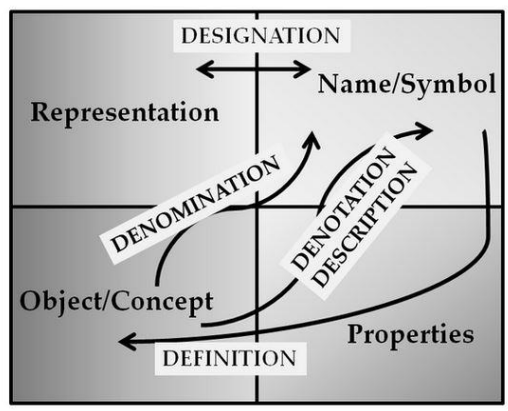

Figure 1.

Our main research hypothesis is that switching from a form of pairing to another one is an important step in the learning process, and in fact it is the result of a conceptual change (diSessa, 2006). We conjecture that for young children the interplay between mathematical objects, names, properties and representations is influenced and framed by the learning environments where the didactic actions develop. In particular we adopt the point of view of Duval following which there are 4 levels of comprehension of a geometrical figure (Duval, 1995 and Duval 1999): the passage from a level to another one is in fact the result of a conceptual change and can possibly be detected through a change in the process of pairing name/object. As pointed out f.i. by Deliyianni et al (2012), perceptual apprehension (which is the first level in Duval's scheme) indicates the ability to name figures and the ability to recognize in the perceived figure several sub-figures.

These changes are in fact a substantial aspect of Fischbein dynamic conceptual/figural (Fischbein, 1993, and Fischbein and Nachlieli, 1998). In fact we may see the progressive evolution of this dynamic through the evolution of the process of the pairing name/object and in particular by pointing out the appearance of the use of properties in this process. In fact, the progressive fusion of conceptual and figural aspects (where "conceptual" includes the individuation and the use of geometrical properties "fusion" includes the insertion of properties into representations) is made explicit by children through the use of language, in a Vygotskian perspecitve (Vygostki, 1978).

Methodology: the use of Comparative Geometry as a different learning environment

According to our theoretical frame, our methodology for intervening into these interplays relies on the use of comparative geometry. The idea of comparative geometry deals with the basic concepts of spherical geometry compared with the corresponding ideas of elementary plane geometry. This approach offers students and teachers the opportunity of learning getting a creative thought discovering a new geometry. Actually sphere is not a foreign object even for a primary school student (as our research confirms) and can allow them to experience some non-Euclidean constructions comparing them with the classic plane constructions. Moreover young students are still not influenced by the rules of Euclidean geometry and this makes their exploring of situations more effective. Comparative geometry activities allow hence the children to deal with geometrical objects in a learning environment where the relations between objects, representations and properties are different from the usual ones, hence implying a restructuration of the interplays between them.

The basic idea is to introduce primary school students some new topics in the traditional method (Euclidean) and translate these concepts to the sphere as closely as possible. In particular we focused our experimentation on the knowledge they had about circles and circumferences.

Exploring spherical geometry requires to draw shapes on a spherical surface because it is not enough to imagine a spherical figure drawn on a plane. Lénárt Sphere kit helped us to create a new learning environment to do geometry using a plastic sphere, markers, a spherical ruler and a spherical compass.

Our hypothesis is that experimenting spherical geometry activities in tandem with plane geometry ones, helps students in better understanding geometrical objects like the circle, well known in the 
Euclidean case, and also heightens students insights into plane geometry. Problems are always posed in parallel in the two environments.

The simplest line in spherical geometry is a great circle, the largest circle that can be drawn on a sphere, for instance the Equator line or the longitudes (on the Earth surface). Nevertheless a great circle can be considered also a "straight line" on the sphere, besides being a circle. Other circles for instance latitudes, excluding the Equator) don't play the same role of "straight lines" on the sphere.

The contrast between planar and spherical geometry offers a great opportunity to arise questions relating the concept of a circle and the relationship between circle and circumference.

Geometric concepts on the sphere, and related activities, are not more difficult than those of plane geometry, even for a primary school student. The main problem they can find is the will to transfer directly geometric properties from plane to sphere, without considering the different geometrical contexts.

\section{Lénárt Spheres}

Lénárt Spheres (Lénárt, 1993 and 1996) are a well-known tool, used for providing a learning environment for comparative geometry activities where the relations between points, circumferences, right angles, properties like minimal distance and so on are different from the usual ones. They are used in all school levels. Even if they are used especially for advanced mathematical learning (in particular for exploring non-Euclidean geometries), these artefacts already proved to be useful in investigating children' actions and their construction of mathematical meanings (Antonini and Maracci, 2013) since the comparison between the geometries may help teachers in creating a-didactic situations, students in getting rid of some mathematics preconceptions and prejudices and both teachers and students in breaking the didactic contract.

The research questions.

1) The word "circle" in familiar to our students, both through school activities and by means of the natural language. What is associated to the word "circle" for our populations?

2) Are there different categories of students with respect to this pairing name/object, in the case of the word "circle"?

3) Can a particular activity in comparative geometry foster a switching from a category to another?

\section{The experimental setup}

Framework of the activities with children

Our planned activities with the Lénárt Spheres were task-designed activities in small cooperative groups. Basic mathematical objects involved in these activities were: the point as the simplest object both on the plane and on the sphere, and recognition of antipodal points; the great circle as the simplest and shortest "line" on a sphere; the role of equators.

In particular, we focused on the description and the construction of the circle on the sphere, by using a spherical compass.

All the activities performed by the groups were recorded and pupils' actions and oral expressions were classified.

The population.

Our population consisted of 5 classes, two of grade 2 in the region of Milan, one of grade 3 in the region of Ravenna and two of grade 4 in Rome and Milan, for a total of 128 students. The percentage of non-native-language students was approximately the $15 \%$. Classes have been individuated after interviews with teachers and headmasters. They were classes of medium-high level, with respect to the average level in Italy, both as socio-economic background, and as learning performances (this 
comparison has been performed via the national standardized test results). Teachers were participating to the activities of a research group in didactic of mathematics.

Experimental tools.

In the classes of grade 3 and 4, two short questionnaires were administered, one before (Q1) and one after (Q2) the activities. These questionnaires were intended to detect and categorize how students associate to the name "circle" an object (mathematical or not) or a class of objects (mathematical or not). In particular, the questions tried to identify the use in this association of concrete models, properties, representations or constructions. The first questions in Q1 asked the pupil to explain to a friend who does not know the meaning of the word "circle", what a circle is. Another question in Q1 asked if they knew the difference between "circle" and "circumference".

In Q1 an item showed an ellipse, a regular 20-gone, a regular 9-gone and a square, asking to check the figure more similar to a circle and to explain the reasons of the choice.

In the classes of grade 2 we developed two collective interviews based on the same tasks proposed in Q1 and Q2. These interviews have been recorded.

\section{The Laboratory activities}

The Laboratory activity has begun by introducing the Lénárt Sphere and leaving students the freedom of exploring them, by manipulating and by drawing on them in order to become familiar with the artefacts. Later they were introduced to some basics of spherical geometry, such as how to consider the "distance" (and in particular the "minimal distance") between two points on the sphere. In order to do this, students had to draw two points randomly on the sphere and to try to connect them freehand in a manner minimizing the distance between them. In support to this activity, a spherical ruler has been provided- students used it in order to verify the actual lengths of the drawn lines. The next step was the introduction of a spherical compass. The discussions between children have been recorded and transcribed.

\section{Results}

We will discuss here in details the results of the two classes of grade 4 (46 students, 9 years old).

\section{Categories}

Three main categories and six sub-categories have been individuated in the answers to Q1, classifying what is associated to the name "circle".

Category S- The name "circle" is associated to the shape. Here the association name/object is completely played in the frame of perceptual apprehension (Duval, 1995 and 1999). With the language of van Hiele levels (van Hiele, 1986; and Crowley, 1987), here we are in the domain of visualization. We classified 26 children in this category. Their answers can subdivided into three categories (note that sometimes a child uses more than one kind of description).

S1. "Circular answers". These descriptions of what a circle is use another formulation of the word "circular", f.i. The circle is a circular figure or The circle is a round figure (sometimes The circle is a round line) or even (with a dimensional jump) The circle is spherical. This is a very early state, there is no explicit association name/object.

S2. "Analogical answers". These descriptions refer to objects whose shape might help the "friend" in understanding what a circle is. Objects frequently referred to have been the planet Earth (surely this is related to some teaching fact or experience, since of course the spherical shape of the Earth is not directly experimented by the children), a soccer ball, street signs, and even an hula-hoop. Hence onedimensional (the hula-hoop), two-dimensional (the street signs, a squeezed ball or a material disk) and three dimensional (the soccer ball,) objects were invoked. A child specified that A circle may be tridimensional. 
This is what D'Amore and Fandiño Pinilla (2012) call denomination (following the schematization of Bolondi et al (2014); these are our references also for the following categories).

S3. "Ostensive answers". These descriptions invoke the possibility of drawing the shape, in order to show with a representation to the friend what a circle is. Other answers evoke the movement of a finger along the border of a circle (sometimes individuating a sort of "homogeneity" of the boundary of a circle: If you follow with your finger the boundary of the circle, you will be always at the same point). We argue that we are in presence of a designation.

Category P- The name "circle" is associated to a figure having a particular property. The association name/object is played in the frame of discursive apprehension, in the sense of Duval as quoted above. In this category we classified 14 children, whose answers can be subdivided into two categories.

P1. "Descriptive answers". These descriptions mainly refer to the facts that

- A circle has no sides, no angles, no vertexes;

- A circle has just one side;

- A circle has no holes and no recesses (i.e., it is a convex figure).

Some answers refer also to the symmetries of a circle. This is a description or a designation.

P2. "Distance answers" (in fact just two children gave this kind of answer in Q1). A child explained in a confuse but substantially correct way that a circle is something determined by a centre and a distance. The other explained that If you make a point in the centre [sic] the distance from the boundary is the same everywhere. We are in presence of a first manifestation of a definition.

Category C- "Constructive answers". The name circle is associated to a particular construction, sometimes with the use of the compass (but not only). This is again a description but of constructive nature. The association name/object is played in the frame of sequential apprehension, in the sense of Duval. We classified in this category 6 children.

Similarity with a circle

In Q1, an item showed 4 figures (an ellipse, a regular 20-gone, a regular 9-gone and a square) asking children to check the figure more similar to a circle, arguing their choice. 42 of them checked the regular 20-gone, and just 4 checked the ellipse. This is quite interesting, since the regular 20-gone was the choice of all the children who stated that $A$ circle has no sides, no angles or no vertexes. On the other hand, three children have justified their choice of the 20-gone with a symmetry argument.

Changes from $Q 1$ to $Q 2$

4 children switched from category $\mathrm{S}$ to category $\mathrm{P}$ in our observation and 4 from category $\mathrm{S}$ to category C. 3 children switched form a description of type S1 to one of type S2. These changes have been detected thanks to the use, in the answers in Q2, of qualitatively different expressions from those used in the corresponding Q1's.

Circle and circumference,

As clearly appeared in Q1, children of one of the two classes were aware of a difference between circumference and circle (21 over 25$)$, whilst in the second one the situation was less clear (only 5 over 21 were able to distinguish between them). In one case, an interesting "physical" distinction was evoked, namely A circumference may be oval, in the sense that it can be squeezed, whilst a circle cannot be squeezed.

In most cases the difference was stated as the circle is a figure, the circumference is its boundary. Hence the difference was made explicit in term of inclusion of one object into the other- one child used the verbal expression A circle has a circumference, and another said A circle may have many circumferences.

Nevertheless as already pointed out, when they were asked to speak about $a$ circle, they indifferently made reference both to the circle and to the circumference. 
This relationship of inclusion might be said a relationship of physical inclusion, which becomes a relation of logical definition between the two. Note that no child in this class pointed out this relationship in the opposite way, i.e. by defining a circle by what is inside a circumference, as it happens in advanced students.

Drawing a circumference on an egg surface: some remarks.

In questionnaire Q2 children were asked to draw a circumference on the surface of an egg, and a picture of a real egg was provided. 12 children over 46 drew boundary circumferences, hence showing a permanence of this physical-strongly related to the perceptive image- relationship. A similar behaviour has been described for the image of a cube by Fischbein (1993).

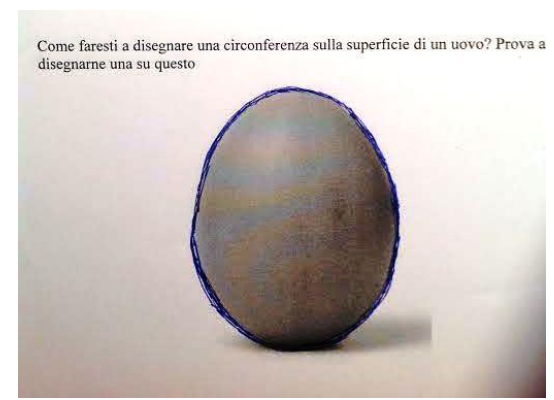

Figure 1. Circumference as boundary of the egg.

Three children drew a northern pole circumference of this kind

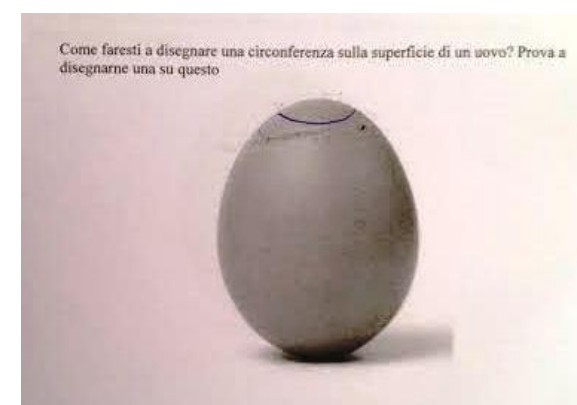

Figure 2. Northern pole circumference.

The majority (15 children) drew a diametral circumference. In the following cases, children were able to imagine the egg and a way to draw a circumference on it, and then to represent it on the picture.

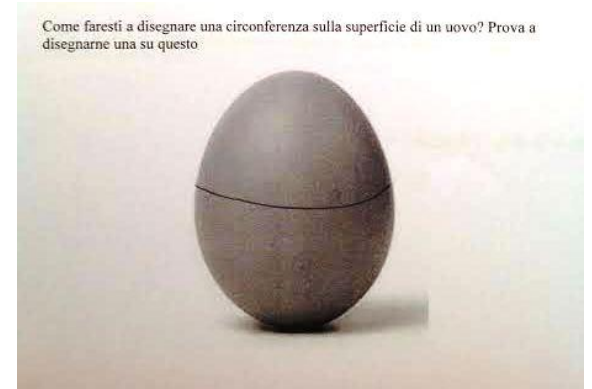

Figure 3. Diametral circumference.

In some cases both kinds of circumferences (diametral and boundary) appeared. 


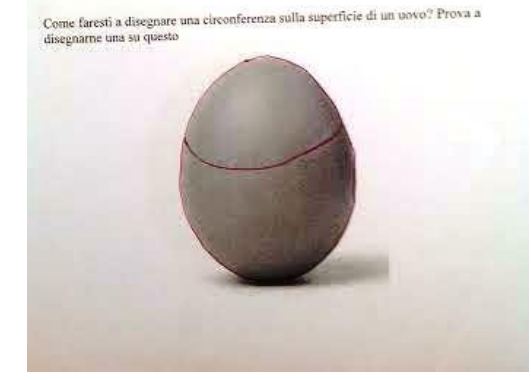

Figure 4. Diametral and boundary circumferences.

As a last possibility, some children (7) drew what we called label circumferences.

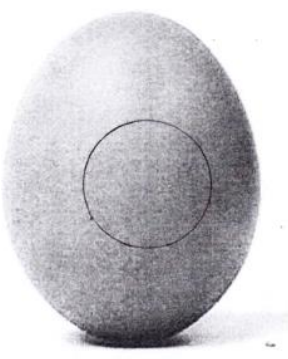

Figure 5. "Label” circumference, 1

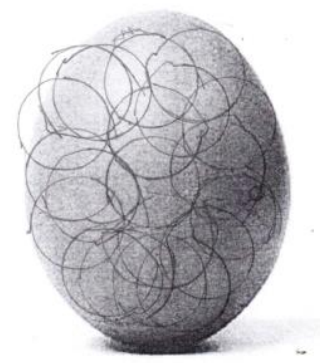

Figure 6. "Label" circumferences, 2

Of course, some children drew many different circumferences, as in the following case where we have both label (1 and 2) and "orbital" (3) circumferences:

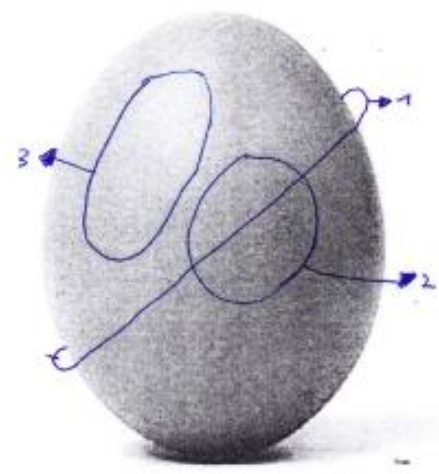

Figure 7. A mixed case 


\section{Conclusions and further perspectives}

We may conclude that students associate a content to the word "circle" in different ways, which refer to different processes in which the interplays between the objects, their properties and their representations may change due to a didactic action. In our population of 9 years old children of grade 4, the perceptual level of apprehension is manifested in the majority of them, and it appears also in their way of describing what a circle is. The use of a particular artefact allowed children to reinvestigated the relationships between the mathematical objects, their representations, their constructions and their properties. After the use of the artefact, 11 children over 46 changed their way of describing what a circle is.

We are using the categories individuated for classifying the behaviours of the whole population of our research, with the aim of mapping through the different school levels the evolution of these processes.

\section{Acknowledgements}

The authors thank Lucia Baldazzi, Claudia Gualandi, Annarita Monaco and Rosalia Tusa for their fundamental help during the experimental phase of this research and especially in collecting protocols.

\section{References}

Antonini, S. and Maracci, M. (2013). Straight on the Sphere: Meanings and Artefacts. In A.M. Lindmeier and A. Heinze (Eds), Proceedings of the 37th Conference of the International Group for the Psychology of Mathematics Education, Vol.2 (pp. 36-40). Kiel, Germany: PME.

Bolondi, G., Ferretti, F. and Maffia, A. (2014). Monomials and Polynomials: the long march towards a definition (to appear)

Crowley, Mary L. (1987). "The van Hiele Model of the Development of Geometric Thought". In Learning and Teaching Geometry, K-12, 1987 Yearbook of the National Council of Teachers of Mathematics, edited by Mary Montgomery Lindquist (pp.1-16). Reston, Va.: National Council of Teachers of Mathematics.

D'Amore, B., and Fandiño Pinilla, M.I. (2012). Su alcune D in didattica della matematica: designazione, denotazione, denominazione, descrizione, definizione, dimostrazione. Riflessioni matematiche e didattiche che possono portare lontano. Bollettino dei docenti di matematica, n.64, 33-46.

Deliyianni, E., Elia, I., Gagatsis, A., Monoyiou, A., and Panaoura, A. (2006). A theoretical model of students'geometrical figure understanding. In V. Durand-Guerrier, S. Soury-Lavergne and F. Arzarello (eds.), Proceedings of CERME 6. Lyon, France: Cerme.

diSessa, A. A. (2006). A history of conceptual change research: Threads and fault lines. In K. Sawye (Ed.), Cambridge Handbook of the learning sciences (pp. 265-281). Cambridge, UK: Cambridge University Press.

Duval, R. (1995). Geometrical Pictures: Kinds of Representation and Specific Processes. In R. Sutherland and J. Mason (eds.), Exploiting mental imagery with computers in mathematical education (pp.142-157). Berlin, DE: Springer.

Duval, R. (1999). Representation, Vision and Visualization: Cognitive Functions in Mathematical Thinking. Basic Issues for learnin., Retrieved from ERIC ED 466379.

Duval, R. (2008). Eight problems for a Semiotic approach in Mathematics Education. In L. Radford, G. Schubring and F. Seeger (Eds.), Semiotic in Mathematics Education (pp.39.61). Rotterdam, NL: Sense Publishers.

Fischbein, E. (1993). The theory of figural concepts. Educational Studies in Mathematics, 24 (2), 139-162.

Fischbein, E., and Nachlieli, T. (1998). Concepts and figures in geometrical reasoning. International Journal of Science Education 20(10), 1193- 1211.

Lénárt, I. (1993). Non-Euclidean Adventures in the Lénàrt Sphere. Emeryville, Ca: Key Curriculum Press.

Lénárt, I. (1996). Alternative models on the drawing ball. Educational Studies in Mathematics, 24, 277-312.

Radford, L. (2003). Gestures, speech, and the sprouting of signs: a semiotic-cultural approach to students' types of generalizatio. Mathematical Thinking and Learning, 5(1), 37-70.

van Hiele, P.M. (1986). Structure and insight. A theory of Mathematics Education, Orlando, Fl: Academic Press.

Vygotsky, L. S. (1978). Mind in Society. The Development of Higher Psychological Processes, Harvard, Ms: Harvard University Press. 